\section{Case Reports in Dermatology}

\title{
Extensive Lepromatous Lymphadenitis Preceding Lesions on the Face and Earlobes: An Unusual Presentation of Leprosy in Singapore
}

\author{
Shi Yao Sam Yang ${ }^{a}$ Wai Mun Sean Leong \\ Cruz Maria Teresa Kasunuran $^{b}$ Jing Xiang Huang ${ }^{c}$ Sue-Ann Ju Ee Ho ${ }^{a}$ \\ Chen Wee Derrick Aw $^{a}$ Reshma A. Merchant ${ }^{b}$ \\ ${ }^{a}$ Division of Dermatology, National University Health Systems, Singapore, Singapore; \\ ${ }^{b}$ Division of General Medicine, National University Health Systems, Singapore, Singapore; \\ ${ }^{c}$ Department of Pathology, National University Health Systems, Singapore, Singapore
}

\section{Keywords}

Lepromatous leprosy · Lepromatous lymphadenitis · Mycobacterium leprae

\begin{abstract}
Leprosy is also known as Hansen disease, as in some countries the diagnosis of leprosy carries a negative stigma and patients fear being shunned as outcasts. Presently, leprosy is primarily limited to specific geographical regions in resource-poor countries. As a result, there is increased difficulty for the younger generation of physicians today to correctly identify leprosy due to a lack of exposure and a low-index of suspicion, particularly in developed countries. In this case, the indurated lesions over the face demonstrated a preference for the outer lateral aspects over the maxillary areas, the nose bridge, and the pinna of the ears consistent with the organism's preference for cooler regions of the body. This was also evident in the other skin lesions affecting the more acral regions of the limbs in the early stage of disease progression. There is a need to keep this infective condition as an alternate diagnosis to all unusual cutaneous lesions.

(C) 2018 The Author(s) Published by S. Karger AG, Basel
\end{abstract}

\section{KARGER}

Shi Yao Sam Yang, MBBS, MRCP (UK)

Division of Dermatology, National University Health Systems

5 Lower Kent Ridge Road

Singapore 119074 (Singapore)

E-Mail sam_yang@nuhs.edu.sg 


\section{Case Presentation}

A 31-year-old Filipino female presented to the emergency department with a high-grade fever over a 2-week period. This was accompanied by tender swellings in the groin bilaterally that resulted in difficulty walking. She was seen by her family practitioner twice during this episode and was prescribed a 1-week course of oral augmentin, followed by another 5day course of oral moxifloxacin. Despite the antibiotics, her condition continued to worsen. Ten days from fever onset, she developed a rash over her hands and legs, associated with circumoral numbness. However, no swallowing problems or breathing difficulty were reported. Over the next 4 days, the rash spread to her face and ears associated with markedly tender swellings in her armpits.

She has no significant medical history and has been working in Singapore as a domestic helper in the same household for the past 8 years. She is single, with no sexual activity nor gynecologic issues. She has not returned to her native country in the past 3 years.

On systemic enquiry, there was no loss of weight or night sweats, no joint swellings or arthralgia, no alopecia, and no dry eyes or mouth. There was also no personal or family history of autoimmune disease (Fig. 1).

On examination, her temperature was $39.2^{\circ} \mathrm{C}$, pulse rate 120 beats per minute, and blood pressure 130/80 mm Hg. The significant findings concerned the skin, which revealed an erythematous papulonodular eruption scattered over the extensor surfaces of both upper and lower limbs. There were multiple indurated plaques on the face over the nasal bridge, the brow, the outer maxillary area and both earlobes and pinna. This was associated with ankle edema, tender, enlarged and matted cervical, axillary, and inguinal lymph nodes. The rest of the physical examination was unremarkable. The differential diagnoses considered included tumid lupus erythematosus, sarcoidosis, cutaneous amyloidosis, lymphoproliferative disease, Sweet syndrome, and Hansen disease.

Routine investigations revealed leukocytosis of $36.18 \times 10^{9} / \mathrm{L}$, an erythrocyte sedimentation rate of $80 \mathrm{~mm} / \mathrm{h}$ and a C-reactive protein level of $159 \mathrm{mg} / \mathrm{L}$. HIV testing, antinuclear antibody, C3, C4, and double-stranded DNA levels were normal. Serum alkaline phosphatase was elevated at $316 \mathrm{IU} / \mathrm{L}$ with otherwise normal liver transaminases. Her albumin level was $34 \mathrm{~g} / \mathrm{L}$.

In view of the persistent high fever and multiple enlarged lymph nodes with elevated serum alkaline phosphatase, she underwent a computed tomography scan of the neck, thorax, abdomen and pelvis, which showed no intra-abdominal or hepatobiliary source of infection, or abscesses, but revealed multiple enlarged and necrotic lymph nodes in the axilla and inguinal regions consistent with the initial examination findings.

Closer examination revealed bilaterally thickened greater auricular nerves and lateral peroneal nerves, which further raised the suspicion of leprosy. Complete examination of the central nervous system did not reveal any sensorimotor loss (Fig. 2).

Skin biopsy was performed and histological examination showed a diffuse inflammatory infiltrate within the dermis and subcutaneous tissue, sparing the epidermis. The inflammatory infiltrate was composed of lymphocytes, histiocytes, foamy macrophages, and neutrophils. Some of the inflammation involved the nerves and adnexal structures. Large numbers of acid-fast bacilli were identified on Fite and Ziehl-Neelsen stains. These features are consistent with the diagnosis of lepromatous leprosy with erythema nodosum leprosum.

She was started on multidrug therapy comprising of rifampicin, dapsone, and doxycycline. Due to the lack of availability of clofazimine, doxycycline was used as an interim third agent before clofazimine was obtained 2 days later. Prednisolone at $1 \mathrm{mg} / \mathrm{kg}$ was also start- 
ed empirically. Of note, there was no evidence of Lucio phenomenon as she did not have skin erosions or ulcers.

She has recovered well on follow-up in the outpatient setting. The skin lesions, peripheral edema, and tender lymphadenopathy have improved.

\section{Discussion}

Leprosy is also known as Hansen disease, as in some countries the diagnosis of leprosy carries a negative stigma and patients fear being shunned as outcasts. It is caused by Mycobacterium leprae, an acid-fast, obligate intracellular bacterium that demonstrates tropism for Schwann cells and macrophages with a predilection for cooler regions of the body [1]. The resultant chronic granulomatous infection can result in permanent neurological impairment ranging from hypo-pigmented lesions with reduced sensation to gross deformity. Other systemic features would include bacillary infiltration of the optic nerve with resultant blindness, nasal mucosa involvement, and testicular infiltration. If untreated, renal involvement and amyloidosis also can occur [2].

Due to a successful leprosy elimination campaign by the World Health Assembly in the 1990s, and the use of multidrug therapy that resolved problems with drug resistance, there has been a corresponding fall in leprosy prevalence [2]. Presently, leprosy is primarily limited to specific geographical regions in resource-poor countries such as, for example, Nepal, Brazil, and Myanmar [2,3].

As a result, there is increased difficulty for the younger generation of physicians today to correctly identify leprosy due to a lack of exposure and a low-index of suspicion, particularly in developed countries. Moreover, leprosy demonstrates a wide range of clinical presentations as classified by Ridley and Jopling, from subclinical infections to hypopigmented anesthetic patches of tuberculoid leprosy (TL), to widespread plaques, deformities, diffuse skin infiltration, and the classical leonine facies in lepromatous leprosy. This is dependent upon the affected individual's cellular immunity and response to infection [4].

Additionally, the dynamic nature of the immune response to this infection also results in changes of the clinical state, which are known as leprosy reactions. It is important that the physicians of today are also able to recognize and manage these clinical manifestations.

Type 1 leprosy reactions, which occurred in this patient, are demonstrated by the presence of peripheral edema and painful inflammation. This is mediated by the infiltration of interferon $\gamma$ and tumor necrosis factor $\alpha$ secreting CD4-positive lymphocytes in the skin and nerves [5]. This usually resolves with steroid therapy.

Type 2 leprosy reactions or erythema nodosum leprosum are characterized by tender papules and nodules with signs of systemic toxicity - fever, malaise, and joint pains [6]. This normally occurs in the early stage of treatment, but can also entail multiple recurrent episodes lasting more than 6 months [7].

Additionally, greater access to healthcare also results in earlier presentation of the underlying disease process, and typical features that tend to develop over time may not yet be present at the time of consultation. As such, there is a need to keep this infective condition as an alternate diagnosis to all unusual cutaneous lesions.

In this case, the indurated lesions over the face demonstrated a preference for the outer lateral aspects over the maxillary areas, the nose bridge, and the pinna of the ears consistent with the organism's preference for cooler regions of the body. This was also evident in the other skin lesions affecting the more acral regions of the limbs in the early stage of disease 
Yang et al.: Extensive Lepromatous Lymphadenitis Preceding Lesions on the Face and Earlobes: An Unusual Presentation of Leprosy in Singapore

progression. Careful examination of the peripheral nerves also demonstrated thickening. The lack of hypoanesthesia over the skin lesions in this particular case was also consistent with this multibacillary form of leprosy which was reflective of reduced cellular immunity to Mycobacterium leprae. Fortunately, the patient responded well to treatment, without any neurological complications.

\section{Statement of Ethics}

The patient provided consent for publication.

\section{Disclosure Statement}

The authors have no conflicts of interest to disclose.

\section{References}

1 Kirchheimer WF, Storrs EE: Attempts to establish the armadillo (Dasypus novemcinctus) as a model for the study of leprosy. I. Report of lepromatoid leprosy in an experimentally infected armadillo. Int J Lepr Other Mycobact Dis 1971;39:693-702.

Britton WJ, Lockwood DN: Leprosy. Lancet 2004;363:1209-1219.

Fine PE: Leprosy: the epidemiology of a slow bacterium. Epidemiol Rev 1982;4:161-188.

Jullien D, Sieling PA, Uyemura K, Mar ND, Rea TH, Modlin RL: IL-15, an immunomodulator of T cell responses in intracellular infection. J Immunol 1997;158:800-806.

5 Khanolkar-Young S, Rayment N, Brickell PM, Katz DR, Vinayakumar S, Colston MJ, et al: Tumour necrosis factor-alpha (TNF-alpha) synthesis is associated with the skin and peripheral nerve pathology of leprosy reversal reactions. Clin Exp Immunol 1995;99:196-202.

-6 Walker SL, Lockwood DN: The clinical and immunological features of leprosy. Br Med Bull 2006;77-78: 103-121.

7 Pocaterra L, Jain S, Reddy R, Muzaffarullah S, Torres O, Suneetha S, et al: Clinical course of erythema nodosum leprosum: an 11-year cohort study in Hyderabad, India. Am J Trop Med Hyg 2006;74:868879. 


\section{Case Reports in Dermatology}

\begin{tabular}{l|l}
\hline Case Rep Dermatol 2018;10:35-40 \\
\hline DOI: $10.1159 / 000462959$ & $\begin{array}{l}\text { ○ 2018 The Author(s). Published by S. Karger AG, Basel } \\
\text { www.karger.com/cde }\end{array}$ \\
\hline
\end{tabular}

Yang et al.: Extensive Lepromatous Lymphadenitis Preceding Lesions on the Face and Earlobes: An Unusual Presentation of Leprosy in Singapore

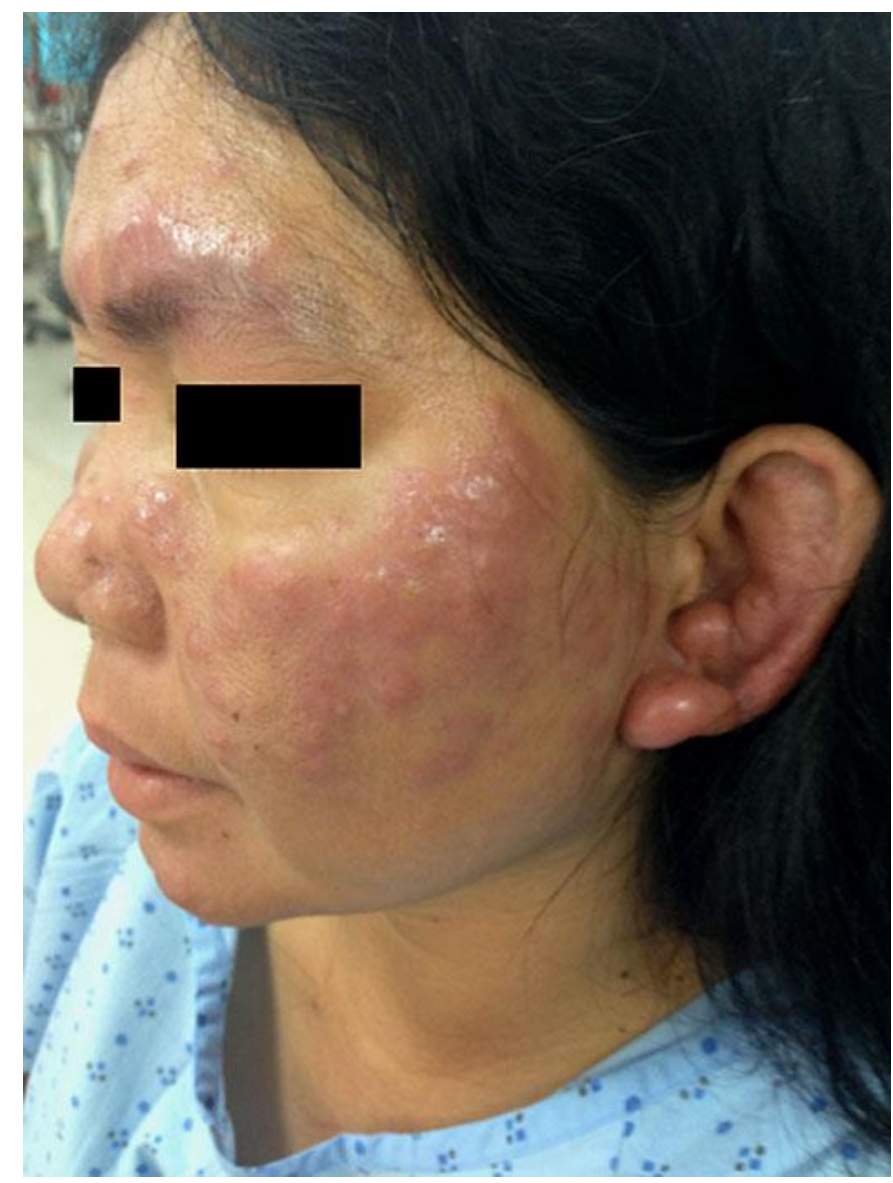

Fig. 1. Multiple indurated plaques over the face, in particular, over the nasal bridge, the brow, the outer maxillary area, and both ears. 


\section{Case Reports in Dermatology}

a
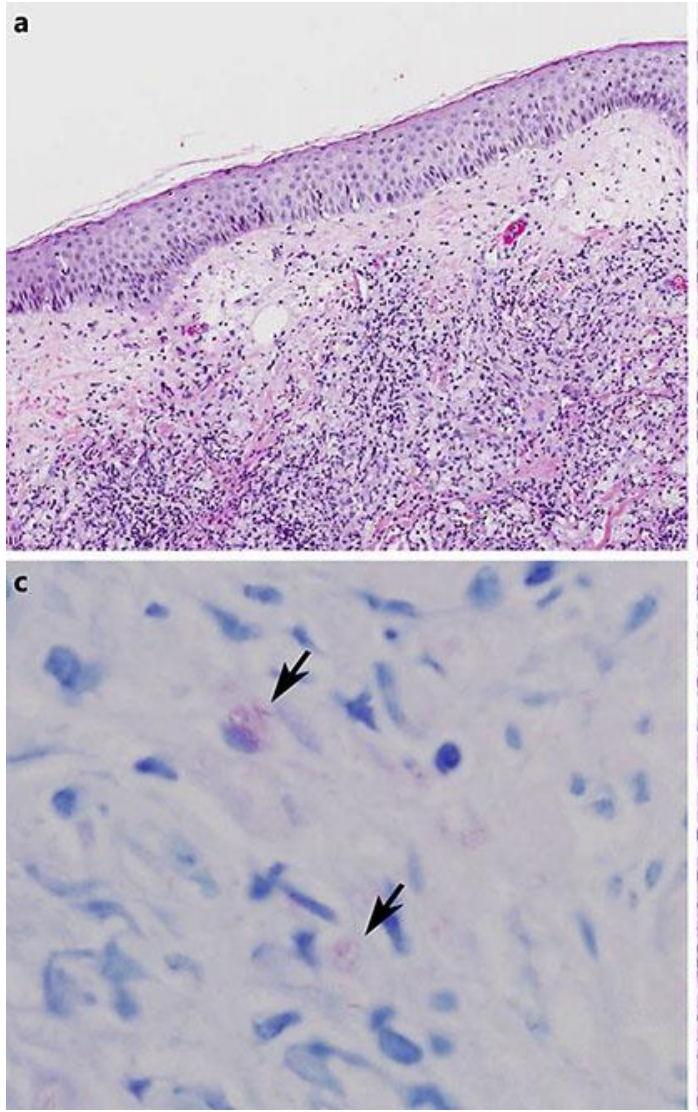
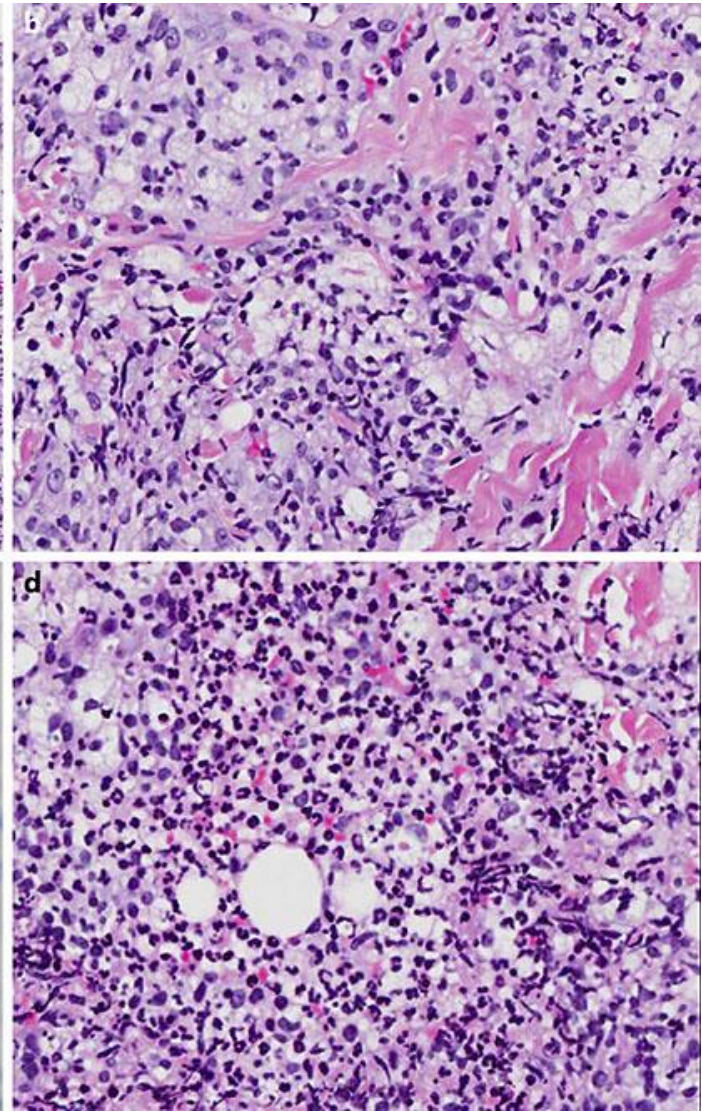

Fig. 2. H\&E-stained section of a skin biopsy showing a dermal inflammatory infiltrate separated from the epidermis by a Grenz zone (a). The inflammatory infiltrate shows abundant foamy macrophages (b) which contain acid-fast bacilli (arrows), identified on Fite stain (c). Subcutaneous tissue showing areas of neutrophilic infiltrate, suggestive of erythema nodosum leprosum (d). 